\title{
Die photosensibilisierende Wirkung verschiedener cancerogener und nicht cancerogener Kohlenwasserstoffe auf die Hefezellen
}

\author{
Von A. Graffi, H. Kriegel, H. Schreiber und F. Windisch \\ Aus dem Institut für Strahlenforschung der Humboldt-Universität zu Berlin, und dem Institut \\ für Medizin und Biologie der Deutschen Akademie der Wissenschaften, Berlin-Buch \\ (Dir.: Prof. Dr. W. Friedri c h) \\ (Z. Naturforschg. 8 b, 142-145 [1953]; eingegangen am 2. Januar 1953)
}

\begin{abstract}
Die photosensibilisierende Wirkung verschiedener cancerogener und nicht cancerogener polycyclischer Kohlenwasserstoffe (3.4-Benzpyren, 9.10-Dimethyl-1.2-benzanthracen, Pyren, Anthracen) auf die Hefezelle wird untersucht.

Es wird gezeigt, daß alle verwendeten Substanzen eine unterschiedliche quantitative photodynamische Wirkung aufweisen.

Die Versuchsergebnisse werden treffertheoretisch ausgewertet. Speziell für das 3.4-Benzpyren resultiert eine völlige Übereinstimmung zwischen der Überlebenskurve und der theoretischen Eintrefferkurve.

Eine Parallelität zwischen cancerogener und photodynamischer Wirksamkeit besteht nicht.
\end{abstract}

$\mathrm{D}^{\mathrm{i}}$ in der experimentellen Krebsforschung verwendeten cancerogenen polycyclischen Kohlenwasserstoffe weisen ausnahmslos bei Bestrahlung mit ultraviolettem Licht eine mehr oder weniger starke Fluoreszenz auf, die meistens im blauen, seltener im blaugrünen Spektralbereich liegt. Es ist wiederholt versucht worden, die Stärke oder die Wellenlänge des Fluoreszenzlichtes in quantitative Beziehungen zur cancerogenen Wirksamkeit zu bringen, doch ist der Beweis hierfür bisher auf experimentellem Wege noch nicht gelungen. Wird das Fluoreszenzvermögen eines cancerogenen Stoffes durch chemische Wirkung aufgehoben, so büßt dieser zugleich auch seine cancerogene Wirksamkeit ein. Lediglich B a u r , Rarei und Gummel ${ }^{1}$ fanden, daß Benzpyren, durch cholestenonsulfosaures Natrium als Lösungsvermittler in eine wäßrige Lösung gebracht, zwar seine Fluoreszenz verliert, jedoch seine cancerogene Wirkung beibehält. Wie fast alle fluoreszierenden Substanzen besitzen auch die cancerogenen Kohlenwasserstoffe die Fähigkeit, im Licht lebende Zellen je nach Belichtungsdauer zu schädigen und abzutöten. Mottram und Doniach² untersuchten die photosensibilisierende Wirkung cancerogener Kohlenwasserstoffe (Benzpyren, Dibenzanthracen)

1 K. H. B a u er, B. R a re i u. H. Gummel, Arch. klin. Chirurg. 193, 499 [1938].

2 J. C. M ottram u. J. D oni a ch, Nature [London] 140, 933 [1937]; Lancet 134, 1156 [1938].

3 M. W o lm a n, Nature [London] 145, 592 [1940].

4 A. G. M at oltsy u. G. F a bian, Nature [London] 158, 877 [1946]. und fanden quantitative Unterschiede. Ähnliche Beobachtungen machten Wolman ${ }^{3}$, Matolts y u. $\mathrm{Fabian}{ }^{4}$ und Graffi ${ }^{5,6}$. Bei den Versuchen von Wolman erwiesen sich einige nicht cancerogene Anthracen-Derivate als unwirksam.

Wir sind damit beschäftigt, an einigen cancerogenen und nicht cancerogenen Kohlenwasserstoffen den photodynamischen Effekt näher zu untersuchen und teilen im folgenden unsere ersten Ergebnisse, über die an anderer Stelle ${ }^{7}$ ausführlich berichtet wird, zunächst in zusammenfassender Darstellung mit.

\section{Methodik}

Als Photosensibilisatoren wurden die cancerogenen Kohlenwasserstoffe 3.4-Benzpyren und 9.10-Dimethyl-1.2benzanthracen (DMBA) sowie die nicht cancerogenen Substanzen Anthracen und Pyren benutzt. Die Auswahl der Kohlenwasserstoffe geschah unter dem Gesichtspunkt, daß sie sämtlich eine intensive Fluoreszenz aufweisen und außerdem sehr rasch in die lebende Zelle eindringen 8 , ohne im Dunkeln bzw. im nicht wirksamen Licht eine feststellbare Schädigung hervorzurufen. Der aufgenommene Kohlenwasserstoff wird intrazellular sehr fest verankert, so daß er durch wässerige Lösungen praktisch nicht mehr auswaschbar ist. Dies bringt den Vorteil mit sich, daß stets mit einer genau definierbaren und leicht bestimmbaren intrazellularen Kohlenwasserstoffkonzentration in einer kohlenwasserstofffreien Suspensionsflüssigkeit experimentiert werden kann.

5 A. Gr a f f i, Z. Krebsforschg. 50, 196 [1940].

6 A. Graffi u. H. M a a s, Arb. staatl. Inst. exp. Therap. Forschungsinst. Chemotherap. Frankfurt a. M. 39, 21 [1940].

7 H. K ri e g e l, Dissertat. Berlin 1952

8 A. Graffi, Z. Krebsforsch. 49, 477 [1939]. 
Als Testobjekt diente Unterhefe Saccharomyces cerevisiae, Stamm U, die für jede neue Versuchsserie unter konstanten Bedingungen eigens herangezüchtet wurde und stets im gleichalterigen Zustand zur Verwendung kam. Der ausgewählte anagglutinierende Hefestamm ${ }^{9}$ ermöglichte, was für die qualitative Auswertung des Bestrahlungseffektes durch Auszählen von Kolonien eine unerläßliche Voraussetzung bildet, die Herstellung von vollkommen homogenen Zellsuspensionen und gewährleistete zugleich durch sein granulareiches Plasma eine ausgiebige Speicherung des Kohlenwasserstoffes. Der Ansatz der frisch geernteten Hefezellen erfolgte in einer 10-proz. GlycerinSerum-Lösung mit einem Gehalt an Kohlenwasserstoffen von 1:8000. Nach 1-stdg. Speicherungsdauer wurde die Suspension zentrifugiert und das abgesetzte Zellmaterial durch mehrmaliges, sorgfältiges Auswaschen von äußerlich anhaftenden Kohlenwasserstoff-Resten befreit. Die fluoreszierenden Substanzen waren dann, wie schon früher Graffi $\mathbf{1 0}$ bei Behandlung von Hefezellen mit Benzpyren fluoreszenzmikroskopisch festgestellt hatte, in den Lipoidgranula quantitativ gespeichert, die nach neuesten Feststellungen 11 mit den von A. M e y e r nachgewiesenen Volutinkörnchen 12 nicht als vollkommen identisch gelten können.

Die Bestrahlung erfolgte hinter 2 mm UG 2 mit dem Licht eines Hg-Hochdruckbrenners (Asta-Quarzbrenner, $220 \mathrm{~V}, 3,7 \mathrm{~A})$. Es wurde also mit UV A und UV B unter den gegebenen Verhältnissen belichtet. Zum Zwecke der Bestrahlung wurden die vorher ausgewaschenen Hefezellen in sterilem Leitungswasser suspendiert und alsdann während Zeiten zwischen 0 und 32 Min. bei möglichst geringer Schichtdicke der Strahlung exponiert. Die gesamte Bestrahlungsanordnung wurde mit einer geeichten Thermosäule nach Moll ausgemessen und ihre Konstanz in energetischer Beziehung laufend mit einem Photoelement kontrolliert. Einer Bestrahlungszeit von 32 Minuten entsprach eine auffallende Strahlungsenergie von $28 \cdot 10^{6} \mathrm{erg} \cdot \mathrm{cm}^{-2}$. Die Auswertung des Bestrahlungseffektes geschah durch Auszählung von Kolonien. Hierzu wurde eine bestimmte Menge der Suspension, mit einem abgemessenen Gelatinequantum vermischt, in Petrischalen ausgegossen und $60 \mathrm{Stdn}$. bei $25^{\circ}$ bebrütet. Die Zahl der nach dieser Zeit mit Lupenvergrößerung erkennbaren makroskopischen Kolonien wurde der Zahl der überlebenden, noch vermehrungsfähigen Hefezellen gleichgesetzt.

$$
\text { Versuchsergebnisse }
$$

In Vorversuchen wurde sichergestellt, daß Hefezellen, die lediglich mit den gelösten Kohlenwasserstoffen behandelt werden, ohne daß eine anschließende Bestrahlung mit wirksamem Licht erfolgt, dadurch keinerlei merkbare Beeinträchtigung ihres Wachstumsvermögens während der Beobachtungszeit

9 F. W in dis c h, Biochem. Z. 246, 332 [1932].

10 A. G r a f f i, Z. Krebsforsch. 52, 234 [1941].

11 Gemeinschaftsarbeit von F. Wind is ch u. D. S tierand sowie von H. Ha ehn, Protoplasma, 1953, im Druck. erfuhren. Auch die mit Glycerin-Serum-Lösung vorbehandelten und anschließend mit UV bestrahlten Zellen zeigten selbst bei der maximalen Bestrahlungsdauer von 32 Min. gegenüber unbestrahlten Zellen in Glycerin-Serum-Lösung keine Verminderung ihrer Wachstumsintensität, ein Ergebnis, das mit den Resultaten von Ehrismann und Noethling ${ }^{13}$ übereinstimmt. Als Vergleichstest diente uns die mit Glycerin-Serum-Lösung vorbehandelte Hefe, so daß auf diese Weise eine möglichenfalls durch das Lösungsgemisch verursachte Zellschädigung, was durch biologische Untersuchungen erst noch näher geprüft werden muß, eliminiert wurde.

Sämtliche untersuchten Kohlenwasserstoffe, sowohl die cancerogenen als auch die nicht cancerogenen ergaben unter unseren Versuchsbedingungen den erwarteten photodynamischen Effekt, der jedoch im einzelnen bei zunehmender Strahlungsdosis charakteristische Unterschiede erkennen ließ. Benzpyren zeigte dabei (s. Abb. 1-4) die stärkste sensibilisierende Wirksamkeit, indem die Zahl der noch vermehrungsfähigen Zellen mit steigender Bestrahlungszeit exponentiell bis auf etwa $2 \%$ bei 32 Min. Bestrahlungsdauer abfiel. Die Halbwertsdosis betrug dabei etwa 2 Minuten. DMBA besaß dagegen eine Halbwertsdosis von etwa 11,7 Min., Anthracen eine solche von etwa 32 Min. und Pyren etwa 19,8 Min. Im Gegensatz zu den anderen Kurven konnte die Úberlebenskurve bei Pyren im linearen Maßstab als eine Gerade dargestellt werden.

\section{Diskussion}

Die Auswertung unserer Ergebnisse muß sich einerseits auf die gefundenen quantitativen Unterschiede zwischen den Wirkungen der einzelnen untersuchten Substanzen erstrecken, die durch die Halbwertsdosis gekennzeichnet sind, und andererseits muß geprüft werden, ob der Kurvenverlauf die Annahme von qualitativen Unterschieden des biophysikalischen Wirkungsmechanismus erforderlich macht oder nicht.

Für die quantitativen Unterschiede sehen wir zwei mögliche Ursachen': Die Speicherung der verschiedenen Kohlenwasserstoffe in den Hefezellen kann in verschiedenem Ausmaße erfolgen. Es dürfte sogar als höchst unwahrscheinlich gelten, daß im Durchschnitt gleiche Substanzmengen je Zelle, trotz gleicher äußerer Vorbedingungen, aufgenommen werden. Sodann

12 A. M e y e r, Die Zelle der Bakterien, Jena 1912.

13 O. Ehrismann u. W. Noethling, Z. Hyg. Infektionskrankh. 113, 597 [1932]. 
können auch die verschiedenen Absorptions- und Emissionsverhältnisse der Substanzen eine Rolle spielen. Zwar besteht im allgemeinen kein Unterschied beim Emissionsspektrum eines fluoreszierenden Stoffes, wenn es von verschiedenen Wellenlängen angeregt wird, sofern diese nur absorbiert werden. Aber die gesamte Fluoreszenzlichtintensität ist selbstver-

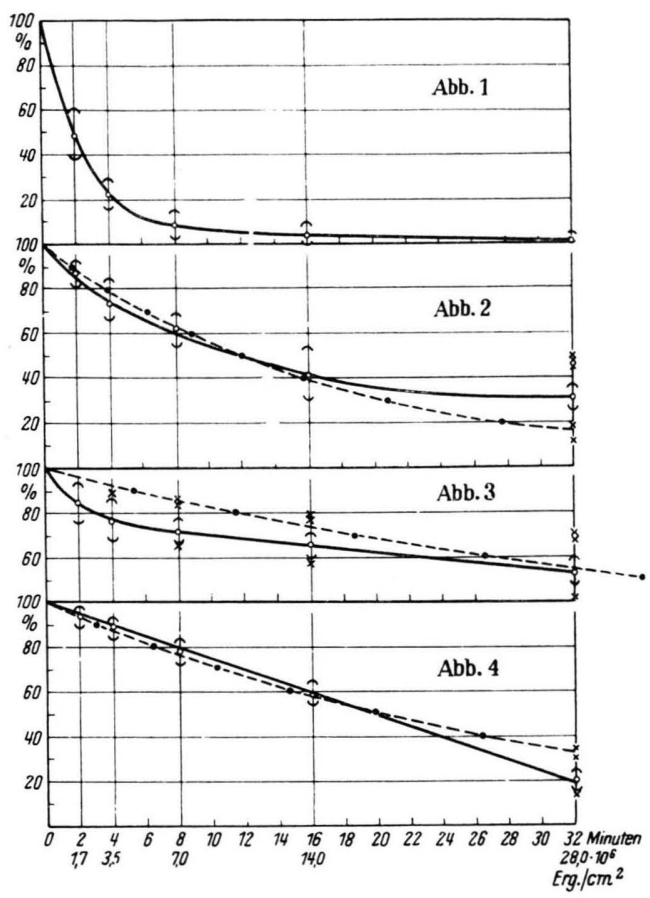

Abb. 1-4. Überlebenskurven der Hefezellen nach Speicherung der Kohlenwasserstoffe und anschließender UVBestrahlung. — experimentelle Kurve, -..... theoretische Ein-Treffer-Kurve. Oo Bereich des dritten mittleren Fehlers, $x$ außerhalb des Fehlerbereichs liegende Prozentwerte.

Abb. 1. 3.4-Benzpyren.

Abb. 2. 9.10-Dimethyl-1.2-benzanthracen. Abb. 3. Pyren.

Abb. 4. Anthracen.

ständlich von der absorbierten Energie abhängig. Diese ist trotz gleicher auffallender Strahlenqualität und -quantität bei den von uns angewandten Substanzen verschieden; denn ihre Absorptionsspektra stimmen nicht überein.

Zur Prüfung der Frage, ob der Kurvenverlauf die Annahme eines prinzipiell unterschiedlichen Wirkungsmechanismus bei den untersuchten Kohlenwasserstoffen nahelegt, wurden die Kurven treffer-

14 L. Herforth, Arch. Geschwulstforsch. 4, 30 [1952].

15 F. H o l w e c k u. A. L a c a s s a g e, C. R. hebd. Séances Acad. Sci. 103, 766 [1930]. theoretisch ausgewertet. In den Abb. $1-4$ ist der Verlauf der theoretischen Ein-Treffer-Kurven in gestrichelter Form wiedergegeben. Bei 3.4-Benzpyren deckt sich die theoretische Kurve mit der experimentellen vollkommen. Aber auch beim DMBA und sogar beim Pyren verläuft die theoretische Kurve innerhalb des Streubereiches der experimentellen Kurven, so daß unsere Versuchsergebnisse mit diesen Substanzen nicht der Annahme eines Ein-Treffer-Vorganges widersprechen. Trotzdem ist natürlich diese unterschiedliche Übereinstimmung zwischen Theorie und Experiment bei den verschiedenen Kohlenwasserstoffen auffällig, was zu der Erwägung berechtigt, daß möglicherweise sekundäre Faktoren wirksam sind.

Zur Aufklärung wurde untersucht, ob etwa die verwendeten Photosensibilisatoren selbst durch die UV-Bestrahlung einen Ab- oder Umbau erleiden. Bei allen von uns überprüften Kohlenwasserstoffen, außer bei DMBA, konnte sowohl in Benzollösung als auch in der Hefezelle bis zur angewandten Maximaldosis nachgewiesen werden, daß dies nicht der Fall ist. Beim DMBA konnten reproduzierbare Meßwerte nicht erhalten werden, was auch schon $\mathrm{H}$ e $\mathrm{r} f$ o $\mathrm{r} \mathrm{h}{ }^{14}$ festgestellt hat. Diese Frage bedarf noch einer näheren Überprüfung. Die Substanzmenge in den Zellen kann also für die ganze Bestrahlungsdauer bei unseren Versuchen als praktisch unverändert und konstant angesehen werden.

Die Tatsache, daß Benzpyren eine wesentlich stärkere photodynamische Wirksamkeit besitzt als DMBA, spricht in gewissem Sinne gegen die Auffassung, daß der photodynamische Effekt auf die toxische Wirkung von Stoffen zurückzuführen ist, die erst unter dem Einfluß der UV-Bestrahlung aus den Photosensibilisatoren entstehen. Auch unser Befund, daß bestrahlte Lösungen der untersuchten Kohlenwasserstoffe keine toxische Wirkung auf nachträglich zugesetzte Hefezellen ausüben - sowohl im unbestrahlten als auch im bestrahlten Zustand -, liegt in der gleichen Richtung.

Sehr auffällig ist der Umstand, daß die gefundenen Überlebenskurven mit Ausnahme des Anthracens innerhalb der Fehlergrenzen mit einer Trefferzahl von $\mathrm{n}=1$ vereinbar erscheinen, während alle bisherigen Überlebensversuche an Hefezellen mit verschiedenen Strahlenarten höhere Trefferzahlen ergaben. So wurde für Röntgenstrahlen die kritische Trefferzahl mit etwa $\mathrm{n}=5-6^{15,16}$, nach anderen

16 R. Glocker, H. Langendorff u. A. Reuß, Strahlentherapie 46, 517 [1933]. 
Autoren mit $\mathrm{n}=2^{17}, 18$ ermittelt. Im ultravioletten Spektralbereich werden je nach der benutzten Wellenlänge verschiedene kritische Trefferzahlen angegeben: für $300 \mathrm{~m} \mu \mathrm{n}=9-40^{15}$; für $253,7 \mathrm{~m} \mu \mathrm{n}=5-6^{19,20}$ bzw. $\mathrm{n}=4^{21}$. Die gleiche kritische Trefferzahl von $\mathrm{n}=4-6$ gilt nach $\mathrm{O}$ s te ${ }^{21}$ auch für die Wellenlängen $222,5,237,8,248,2,265,2$ und $280,4 \mathrm{~m} \mu$. Frilley und Latarjet ${ }^{17}$ fanden für $253,7 \mathrm{~m} \mu$ $\mathrm{n}=10-12$. Für $229 \mathrm{~m} \mu$ wurde von $\mathrm{S}$ chreiber ${ }^{19}$ $\mathrm{n}=29$ gefunden.

Es muß sich demnach bei dem photodynamischen Effekt und bei der direkten Hefeabtötung durch Strahlung um verschiedene, nicht unmittelbar miteinander vergleichbare Wirkungsmechanismen handeln. Darauf deutet auch der Umstand hin, daß der photodynamische Effekt, im Gegensatz zur direkten Strahlenwirkung, konditional an genügenden Sauerstoffzutritt gebunden ist ${ }^{22}$, wonach es auch wahrscheinlich wird, daß an dem photodynamischen Wirkungsmechanismus irgendwie ein Oxydationsprozeß - zumindest als Teilvorgang - beteiligt ist. K a u ts ky ${ }^{23}$ vertritt die Ansicht, daß der Sauerstoff dabei in eine aktive Form gebracht wird. In diesem

17 M. Frille y u. R. L a t a r j et, C. R. hebd. Séances Acad. Sci. 218, 480 [1944].

18 R. L a t a r j et, Ann. Inst. Pasteur 70, 277 [1944].

19 H. S chrei ber, Strahlentherapie 49, 541 [1934]. [1938].
Sinne sprechen möglichenfalls auch die Untersuchungen von $\mathrm{Calcutt}$ und $\mathrm{Newhouse}{ }^{24}$, die durch Zusatz von Cystein-hydrochlorid die photosensibilisierende Wirkung des Benzpyrens herabsetzen konnten. Wir können an Hand der bisher uns vorliegenden Untersuchungsergebnisse zu solchen spezifizierten Fragen noch nicht Stellung nehmen. Durch Abänderung der physikalischen, biologischen und chemischen Versuchsbedingungen erscheint es uns jedoch möglich, zu einer weitgehenderen Analyse des photodynamischen Effektes zu gelangen.

Abschließend sei noch auf unseren Befund hingewiesen, daß die Versuche keine Parallelität zwischen cancerogener und photodynamischer Wirksamkeit ergeben haben. Benzpyren ist photodynamisch wesentlich wirksamer als DMBA, während die beiden Substanzen sich in ihrer cancerogenen Wirkung gerade umgekehrt verhalten.

An dieser Stelle möchten wir den technischen Assistentinnen, Frl. I. Guse und Frl. Ch. Goslich, für ihre Hilfe bei der experimentellen Durchführung der Untersuchungen unseren Dank ausprechen.

21 R. H. Oster u. W. A. Arnold, J. gen. Physiol. 18, 351 [1935].

22 K. N o a c k, Z. Botanik 12, 273 [1920]; 17, 481 [1925].

23 H. K a u t sky, Naturwiss. 19, 1043 [1931]; Ber. Dtsch. chem. Ges. 65, 1762 [1932].

${ }^{24}$ G. Calcutt u. P. Newhouse, Nature [London] 161, 53 [1948].

\title{
Wirkungen von monomerem Acrylamid auf Proteine*
}

\author{
Von Hermann Druckrey, Ursula Consbruch und Dietrich Schmähl \\ Aus der Chirurgischen Universitätsklinik in Freiburg (Direktor: Prof. H. Krauss) \\ (Z. Naturforschg. 8 b, 145-150 [1953]; eingegangen am 13. Dezember 1952)
}

Herrn Prof. Dr. Dr. h. c. A. Buten andt zum 50. Geburtstag

Sole von Proteinen erstarren mit monomerem Acrylamid zu relativ festen Gelen. Die Geschwindigkeit des Vorgangs hängt von der Konzentration beider Partner und von der Temperatur ab. Eiklar, Fibrinogen oder $\gamma$-Globulin erstarren mit Acrylamid 10-1000-fach schneller als Albumine. Die Unterschiede werden in erster Linie auf die Form der Proteine bezogen, die bei den schnell erstarrenden Eiweißkörpern gestreckt oder fadenförmig, bei Albuminen dagegen eher kugelig ist.

Einige Tage nach der Erstarrung wird die Ninhydrin-Reaktion bei den Gelen negativ.

Die Erstarrungsprodukte werden durch Pepsin nicht gespalten, dagegen leicht durch Wasserstoffperoxyd.

Durch Trichloressigsäure läßt sich die Polymerisation von Acrylamid in wäßriger Lösung in Gang setzen.

$\mathrm{D}_{\mathrm{s}}^{\mathrm{is}}$ e Beobachtung, daß bi- oder polyfunktionelle Substanzen starke pharmakologische Wirkungen haben ${ }^{1}$ und sogar Krebs erzeugen können ${ }^{2}$, veranlaßte uns, die Frage zu untersuchen, ob zwischen solchen Substanzen und Proteinen Wechselwirkungen nachweisbar sind. Wegen der Neuartigkeit und

* Die Untersuchungen wurden durch die D e u t s c h e Fors chungs gemeins chaft ermöglicht. 